\title{
Efficient resource allocation method for hitless redundancy switching technology at multiple sites
}

\author{
Go Yazawa ${ }^{1}$, a), Takahiro Kubo ${ }^{1}$, Natsuki Yasuhara ${ }^{1}$, \\ and Shinichi Yoshihara ${ }^{1}$ \\ ${ }^{1}$ NTT Access Network Service Systems Laboratories, NTT Corporation, \\ 3-9-11 Midori-cho, Musashino-shi, Tokyo 180-8585, Japan \\ a) go.yazawa.tw @hco.ntt.co.jp
}

\begin{abstract}
For transmitting video or financial transaction information, communication interruption must be short, so hitless redundancy switching (HRS) technology is a useful solution. However, when this technology is used in multiple sites, a large amount of resources is required for HRS devices to communicate. In this paper, we propose an efficient resource allocation method and demonstrate that it can reduce resource usage by $97 \%$ in a numerical simulation.
\end{abstract}

Keywords: hitless, redundant, switching, Ethernet

Classification: Network System

\section{References}

[1] ITU-T Y. 1541, "Network performance objectives for IP-based services," Dec. 2011.

[2] IEEE 802.1CB, "IEEE Standard for local and metropolitan area networks-Frame replication and elimination for reliability," Oct. 2017. DOI: 10.1109/IEEESTD. 2017.8091139

[3] SMPTE ST 2022-7:2019, "Seamless protection switching of RTP datagrams," May 2019. DOI: 10.5594/SMPTE.ST2022-7.2019

[4] Y. Sakagami, S. Taniguchi, and R. Kawate, "A study and evaluation on hitless switching scheme for dedicated Ethernet," IEICE Technical Report, CS2013-1, pp. 1-5, April 2013.

\section{Introduction}

Wide-area Ethernet has become a useful option for low-cost communication between multiple locations. In wide-area Ethernet services, the network often has redundancy, but communication interruptions due to frame loss occur at redundancy switching, making it difficult to meet the needs of users who require communications with short interruption times, such as transmission of video [1] and financial transaction information. 
Hitless redundancy switching (HRS) technology [2,3] provides communication with no communication interruption time. In this technology, a signal is duplicated at the sending site of hitless switching devices, transferred independently to two paths in the network, and either of the two signals received by the receiving side hitless switching device is selected. HRS technology requires resources such as sequence numbers to keep track of the reception status of each duplicated signal and buffers to wait for processing. The communication between two sites requires one set of resources such as sequence numbers and buffers, while communication between multiple sites requires more sets of resources as the number of sites increases [4].

In a steady state, only appropriate devices receive signals due to media access control (MAC) address learning in Ethernet, so the resource consumption is suppressed to some extent. However, when MAC address learning information is flushed due to route switching in the network, flooding can temporarily consume a large amount of resources and cause them to run out. To prevent the exhaustion of resources, a method needs to be developed to allocate the resources appropriately even during flooding. In this paper, we propose a novel resource allocation method and demonstrate it reduces resource usage in a numerical simulation.

\section{Hitless redundancy switching technology between multiple sites}

\subsection{Principle}

Figure 1(a) shows a conceptual diagram of HRS technology between two sites. The signal sent from customer device $\mathrm{X}$ is assigned a sequence number at HRS device A, duplicated into two, and then forwarded to the two paths in the network. At HRS device $\mathrm{B}$, one of the two received signals is selected and forwarded to customer device Y. To understand the extent to which frames have been sent and received between devices $\mathrm{A}$ and $\mathrm{B}$, device $\mathrm{A}$ assigns a sequence number to the duplicated signal. When a frame with a number different from the expected number arrives, it is buffered until the frame with the expected number arrives, thereby enabling transmission without skipping frame order. By using HRS technology, if there is a communication break in either path, device B on the receiving side can switch the signal path with no communication break and perform HRS.

Figure 1(b) shows a conceptual diagram when HRS technology between two cites is used at multiple sites. In this case, signals communicated from customer device $\mathrm{X}$ to customer devices $\mathrm{Y}$ and $\mathrm{Z}$ are mixed up and transferred to HRS device $\mathrm{A}$. Since device A assigns sequence numbers to the mixed signals, HRS devices B and $\mathrm{C}$ receive the signals with some sequence numbers missing, so the sequence numbers cannot be managed correctly and redundancy switching cannot be performed properly.

Figure 1(c) shows a conceptual diagram of HRS technology between multiple sites, where the concept of traffic flow is introduced to solve the problem above. Here, the traffic flow is defined as a unit that logically classifies signals in the sending and receiving sites of the HRS devices. By classifying each signal to the traffic flows, signals that are mixed during transmission can be logically separated, and sequence numbers are processed appropriately. The traffic flow can be classified by the information contained in the signals such as a combination of source and 


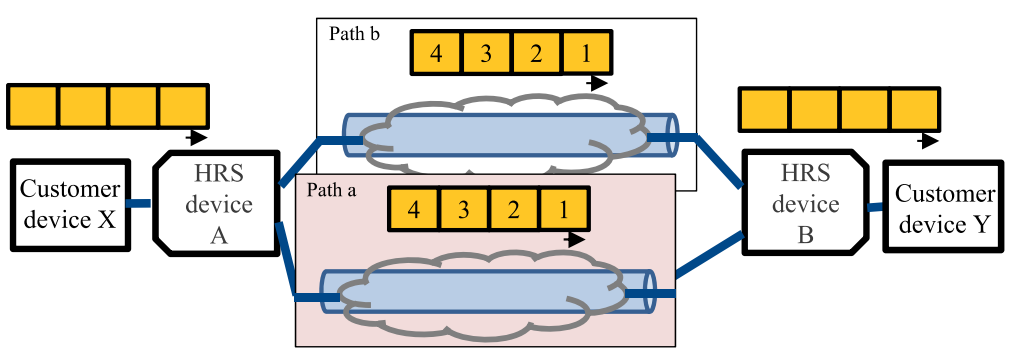

(a) HRS technology between two sites

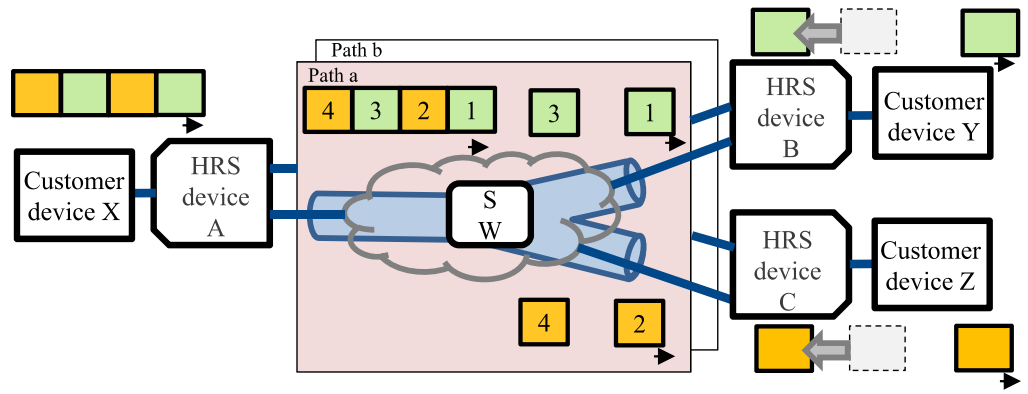

(b) HRS technology between multiple sites w/o introducing concept of traffic flow

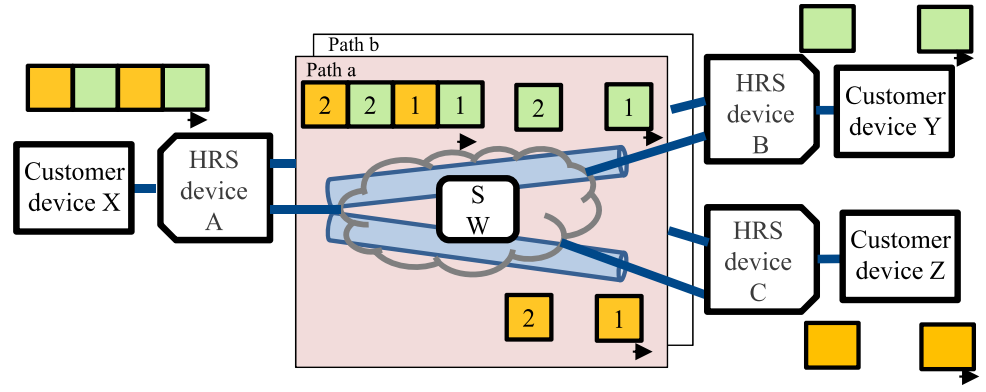

(c) HRS technology between multiple sites w/ introducing concept of traffic flow

Fig. 1. Conceptual diagram of the hitless redundancy switching (HRS) technology.

destination addresses.

\subsection{Estimation of amounts of resources}

When this technology is used, large amounts of resources, such as sequence number and buffers, are required for each HRS device, so we estimate the amount of resources. When the number of sites where HRS devices are deployed is $n$ and each site is connected with mesh topology network, there is a maximum of ${ }_{n} \mathrm{P}_{2}$ traffic flow, and the order of the amount of resources required can be expressed as $O\left(n^{2}\right)$. As the number of sites increases, larger amounts of resources are required.

\subsection{Issues}

In a steady state, only appropriate HRS devices receive signals due to MAC address learning in Ethernet, so resource consumption is suppressed to some extent. However, when the MAC address learning information is flushed due to a route switch of the network, flooding may temporarily consume a large amount of resources and 
deplete them. To resolve resource depletion quickly, we focused on changing the aging time.

Typically, in HRS technology, the aging time should be set to a long value to prevent accidental aging of the resources of communications that are continuously transmitted at regular intervals. This can ensure the continuity of communication. However, in multiple-site communication, resources are kept for a long time even if they are temporarily increased by flooding, so resource allocation for new communication cannot be started promptly during flooding. To solve this problem, the aging time can be shortened. This enables us to release unnecessary resources as soon as possible after flooding is completed, so that we can start allocating resources for new communications as soon as possible. On the other hand, there may be an increased risk of accidentally releasing necessary resources during regular communication. In other words, when the aging time is set uniformly, it is difficult to both reduce the waiting time for new communications during flooding and continue communications that should be performed.

\section{Novel resource allocation method for hitless redundancy switching technology between multiple sites}

To solve this problem, we propose setting different aging times depending on the amount of allocated resources at the time of allocation.

When flooding is not occurring, a long aging time is set to prevent accidental aging of the communication that is originally continuing. When flooding is occurring, a short aging time is set to reduce the waiting time for new communication by releasing resources early after flooding ends. This allocation method makes it possible to both shorten the waiting time and continue communications that should be communicated.

\section{Numerical simulation}

We conducted numerical simulations to evaluate the resource reduction effect of the proposed method.

\subsection{Formularization}

The received traffic flows are classified into four categories. Here, we set the amount of traffic flow that should be received and is allocated at time $t$ as $S a(t)$, the amount of traffic flow that should be received but is not allocated as $S b(t)$, the amount of traffic flow that is received and allocated only during flooding as $T a(t)$, and the amount of traffic flow that is not allocated as $T b(t)$. When the aging time is set as $T$, the probability of keeping the resource at each aging time is set to $A$, the cumulative distribution function of the gamma distribution is set at $f(t)$, the limit of the amount of resources kept in HRS devices is set to $R$, and the allocated traffic flow at time $t$ during flooding can be formulated as follows.

$$
\begin{aligned}
& S a(t)=A S a(t-T)+(1-A)\{S a(t-T)+T a(t-T)\} \frac{S b(t-T)}{S b(t-T)+T b(t-T)} \\
& T a(t)=A T a(t-T)+(1-A)\{S a(t-T)+T a(t-T)\} \frac{T b(t-T)}{S b(t-T)+T b(t-T)}
\end{aligned}
$$




$$
\begin{gathered}
S a(t)+S b(t)=\{S a(0)+S b(0)\}=\text { const } \\
T a(t)+T b(t)=\{T a(0)+T b(0)\}(1-f(t)) \\
S a(t)+T a(t)=R
\end{gathered}
$$

The parameter for the number of HRS devices, $k$, is set to 64 . Then, at the flooding starts $(t=0), S a(0)+S b(0)$ is set to ${ }_{k-1} \mathrm{C}_{1}=63$ and all of these traffic flows are assumed to be allocated, so $S a(0)=63$ and $S b(0)=0$. At $t=0$, in addition to the above, we assume that an extra traffic flow $\operatorname{Ta}(0)+T b(0)={ }_{k-1} \mathrm{P}_{2}=3906$ is received as traffic flow between other HRS devices with flooding.

The cumulative distribution function of the frame receiving interval and flooding duration time are shown in Fig. 2(a). Each receiving interval and flooding duration time are assumed to be a gamma distributed function, with $\mu=200 \mu \mathrm{s}, n=15$, and $\mu=200 \mu \mathrm{s}, n=5$, respectively. By reading the value of the cumulative distribution of receiving intervals for aging time of $T=2.4$ and $1000 \mathrm{~ms}$, keeping probability $A$ can be obtained as $22.8 \%$ and $100.0 \%$, respectively.

Figure 2(b) shows the traffic flows as the times elapsed in the flooding. As you can see, $T a(t)+T b(t)$ decreases as the flooding finishes but $S a(t)+S b(t)$ is constant as $t$.

\subsection{Evaluation}

We first evaluate the conventional method that sets a uniform aging time.

Figure 3(a) shows the ratio of the allocation rate of the traffic flow that has already started communicating at $t=0$ (hereinafter referred to as the existing traffic flow) to the time elapsed. The resource upper bound $R=128,1984,3841$, and the aging time $T=2.4$ and $1000 \mathrm{~ms}$. In the short aging time, the transmittance decreases due to the large value of $A$ for all the $R$ values. However, in the long aging time, the transmittance is almost $100 \%$ regardless of the $R$ value. This indicates that a long aging time needs to be set to keep the transmittance close to $100 \%$.

Figure 3(b) shows the time course of the allocation rate for new traffic flows that occur during flooding. The resources allocated to the extra traffic flow are released after the aging time has elapsed, and the allocation to the new communication starts.

Next, the proposed method is evaluated where the aging time is changed in accordance with the allocated resources amount.

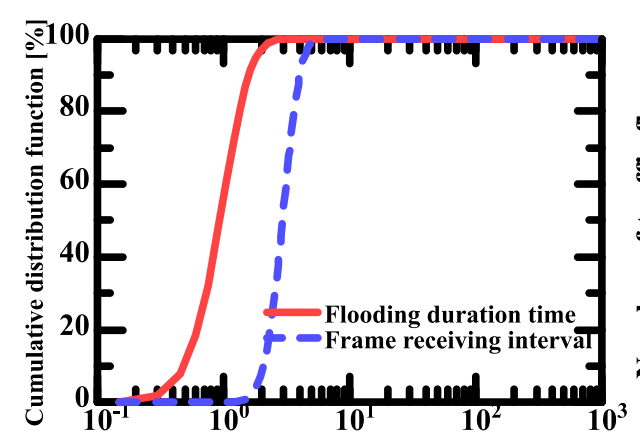

Time [msec]

(a) Cumulative Distribution function of flooding duration time and frame receiving interval.

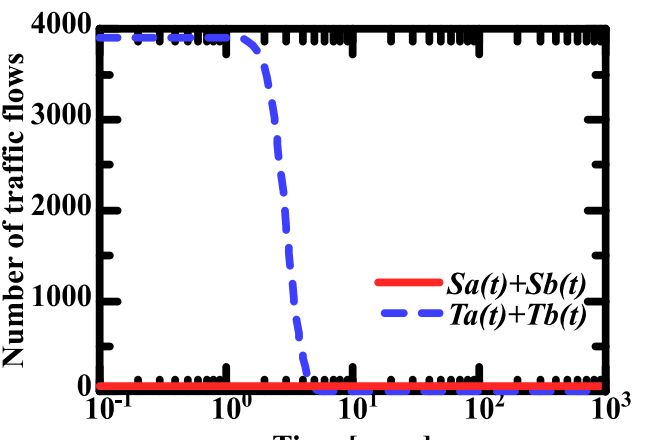

Time [msec]

(b)Number of traffic flows as time elasped.

Fig. 2. Traffic parameters applied in numerical simulation. 


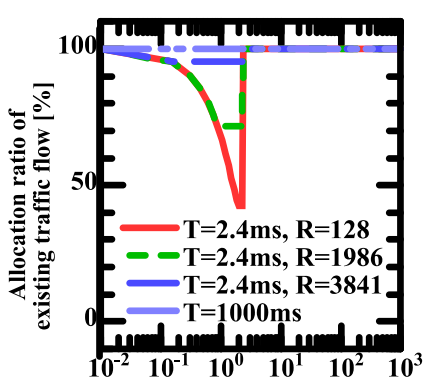

Time [msec]

(a) Time deviation of allocation ratio of existing traffic flow with conventional method.

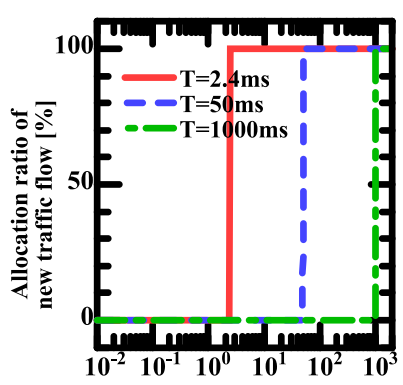

Time [msec]

(b) Time deviation of allocation ratio of new traffic flow with conventional method.

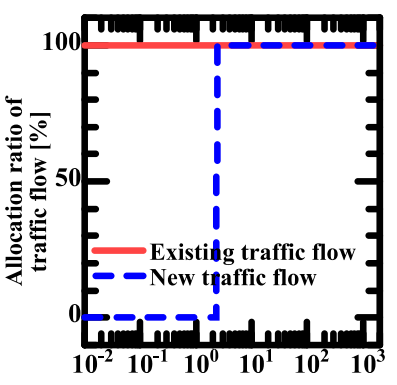

Time [msec]

(c) Time deviation of allocation ratio of existing and new traffic flow with proposal method.

Fig. 3. Time deviation of allocation ratio of existing and new traffic flow with conventional and proposed methods.

Figure 3(c) shows the time variation of the allocation ratio of existing and new traffic flows in the proposed method. In this calculation, the short and long aging times are set to 2.4 and $1000 \mathrm{~ms}$, respectively. The graph shows that by varying the aging time in accordance with the amount of traffic flow, the allocation rate for existing traffic flow can be maintained at $100 \%$, and the allocation for new traffic flow can be implemented earlier.

Results show that the communication can be performed with an appropriate transmission rate even if the resource upper limit $R$ was set to 128 , which is $3 \%$ of the maximum received traffic flow 3969, and that the proposed method reduces resource usage sufficiently by $97 \%$.

\section{Conclusion}

We proposed a new resource allocation method to provide economical network services to users who want to communicate between multiple sites with a shorter uninterruptible time than existing services and demonstrated that it effectively reduces resource usage in a numerical simulation. 